\title{
Defense Information - Semantic Aspects
}

\author{
Rusi Marinov \\ New Bulgarian University, Sofia

\section{Информационна защита- семантични аспекти}

\author{
Руси Маринов
}

Нов български университет, София

\begin{abstract}
Author Note
Rusi Marinov https://orcid.org/0000-0001-6537-1070

Prof.Rusi Marinov,D.Sc., part-time lecturer at the New Bulgarian University.

The author has no conflict of interest to disclose.

Correspondence concerning this article should be addressed to Rusi Marinov
\end{abstract}

New Bulgarian University, 1618 Sofia, Montevideo str.21 Email: rmarinov@nbu.bg

\section{Бележки за автора}

Руси Маринов ID https://orcid.org/0000-0001-6537-1070

Руси Маринов е професор, доктор на науките, хоноруван преподавател в Нов български университет.

Няма конфликт на интереси.

Кореспонденцията, свързана с тази статия, трябва да бъде адресирана до Руси Маринов 1618 Sofia, ул. Монтевидео, 21. Email: rmarinov@nbu.bg 


\begin{abstract}
The study focuses on the semantic aspects of information, the role of cognitive technologies in the time of information defense, the emergence of new concepts related to information security and protection, cybersecurity, and resilience. It can be argued that information is a complex quantity; measuring information means measuring complexity. Artificial intelligence and problem areas related to this area are also the subject of analysis. We are also looking for an answer to the link between cybernetics and cyber technology and to the popular term "cyber" that has recently become buzzword. Another aspect of the study is various models of data structuring and information protection from the point of view of modern strategies.
\end{abstract}

Keywords: semantic information, information defense, cognitive technologies, cybernetics, cybersecurity, cyber, AI, cognitive phenomenon

\title{
Резюме
}

В студията се акцентира на семантичните аспекти на информацията, ролята на когнитивните технологии при защита на информационните активи, появата на нови понятия, свързани със сигурността и защита на информацията, кибер сигурност и устойчивост. Може да се твърди, че информацията е сложна величина, измерване на информацията означава измерване на комплексността. Изкуствен интелект и проблемни области, свързани с това направление са също обект на анализ. Търси се още отговор, каква е връзката между кибернетиката и кибернетичните технологии, респективно популярното понятие „кибер“, превърнало се напоследък в buzzwords. Друг аспект на изследване са различните модели за структуриране на данните и защита на информацията, от гледна точка на съвременните стратегии.

Ключови думи: семантична информация, защита на информацията, когнитивни технологии, кибернетиката, киберсигурност, кибер, изкуствен интелект, когнитивни феномени 


\section{Информационна защита- семантични аспекти}

\section{Въведение}

Терминологията в сферата на защита на информацията е свързана с особена оригиналност, като търпи сериозно развитие в последните 20 години. Появиха са термини като информационна война, мрежова война, кибер война, кибер кризи и киберзащита, хибридна и виртуална война. Тези изрази, от гледна точка на теорията в областта на информационните науки, кибернетика и наука за мрежата, нямат голяма стойност, но от практическа точка навлязоха широко в понятийния апарат на политици, администратори, военни и беше прието съответстващо законодателство. Най-общо казано кибер-технологии представляват усъвършенстване и приложение на науката за кибернетиката в практиката. В средата на 20. век и в началото на 21. век се появяват множество интелигентни програми, роботизирани обекти, които са базирани на основните принципи на кибернетиката. Преди няколко години се появи термина „кибер кризисен мениджмънт“, като в момента съществуват програми и стратегии в тази сфера, но от семантична гледна точка имат относително слаба връзка с кибернетиката. Каква е всъщност същността на кибернетичната теория. Терминът кибернетика като наука за комуникацията и контрола на машините и живите организми произхожда от гръцки "kybernetes"-изкуство за управление на кораб. Самото понятие се превръща в последните години в buzzwords като се използва се за обозначаване на различни процеси. По своя си произход терминът няма отношение към сигурността. Тази дума не е известна до 1948г., когато известният американски учен Норбърт Винер (Wiener,1948/1965). публикува свой труд наречена "Кибернетика". Моделът на кибернетична му структура включва три основни елемента: система; външна среда; негативна обратна връзка. По време на 2-та Световна война Винер разработва комуникационна теория и формулира принципите на кибернетиката. След войната събира група от млади изследователи, специализиращи в областите на изкуствен интелект, компютърни науки и невропсихология и развива и теория, свързана с комуникационното инженерство. През 1943 г. развива основите на кибернетиката, роботиката, компютърен контрол и автоматика (Wiener, 1965). През 1947 г. популяризира името "Cybernetics" и формулира стандартна терминология в тази област. Става първият учен в света, който се занимава с електронна комуникация и контрол (Wiener,1965). Кибернетиката, според сайта на «Американската общност по кибернетика" е ориентирана към разработване на базови теории за комуникация и контрол в комплексни системи (ASC). Приложението на кибернетиката обхваща области като информационни науки, естествени и социални науки, 
образование и мениджмънт. Кибернетиката има два приоритета: изследва потока от информация в определена система; анализ на използване на информацията за самоконтрол. Целта на кибернетиката е да развива език и техники, които позволяват на хората да решават проблеми, с помощта на контрол и комуникация. Тази технология още позволява да се комбинират и класифицират идеи и техники, водещи до организиране на средата. Обратната връзка в кибернетиката означава- информация, относно промените във вътрешната и външна среда, водеща до промяна на системата и адаптацията към средата. Съществуват два типа обратна връзка: негативна-реагира на промяната в средата; позитивна-засилва промяната.

Полезната информация може да включва три важни части: синтактична част, семантична и прагматична. От тази гледна точка се структурират информационните активи в една организация, обект на защита от външно въздействие. От друга страна, ако вземем предвид схващанията в информационните науки нивата на информация могат да се класифицират по следния начин: статистическа: най ниско равнище на информация, единственото, което се изисква, е да знаеш да изчисляваш; синтактично: информацията е структурирана, базирана е на определени правила; има начина да бъде записана, преди да се запише обаче, е необходимо да се усвоят правилата на синтаксиса; семантична: включва значение на информацията, т.е. този тип е н е само структурирана информация, но има значение и е по комплексна величина; прагматична: ориентирана към изпълнение на действия, които кореспондират със значението на информацията, цялата прагматична информация, която съществува в света, е създадена от човека; апробатична: има отношение към поставените цели и е ориентирана към постигане ефектите от информацията. Може да се твърди, че информацията е комплексна величина, измерване на информацията означава измерване на комплексността. Колкото е по сложен проблемът, който изследваме, толкова повече време и пространство се изисква, за да въведем алгоритьм, необходим за решаване на проблема. Специфичната и неспецифичната информация могат да бъдат дефинирани по следния начин: актуализацията на една възможност е специфична, възможността зависи от използваните образци. Неспецифичната информация е противоположно състояние на специфичната. Например, при трансмисия на кодирани сигнали по дадена криптираща система се формира неспецифична информация, след разбиването на крипто системата предаваната информация става специфична. Семантиката включва и понятия като обектна информация или още наречена онтологична, като подобен тип информация има отношение към определен обект и се дефинира като "мрежа от състояния където съществува този обект и структура/характеристики които варират при това състояние. Онтологичната 
информация се дефинира от обекта и няма отношение към субекта, като обектна и онтологична информация се използват паралелно.

Експертите, в частност Томас Дейвънпорт (1997), предлага екологична рамка, която да дефинира всички процеси, свързани с управление на информацията, тази дейност досега беше прерогатив на СIO и служителите по информационни технологии, проблемът е, че тази група експерти, не са подготвени в достатъчна степен, за да направят компетентна преценка на същността на информацията (р. 6-15) От тази гледна точка е необходима рамка, където ясно да направи разграничение между три групи понятия: информация, данни и знание. Възприемането на екологичния подход води до разбирането, че: информацията не може да се съхранява лесно на компютър или в мрежа-това не са данни; колкото по-комплексен е един информационен модел-толкова по-малко полезен може да бъде за бизнеса; информацията може да придобие множество значения в една организация; технологиите са само един от компонентите на информационната среда и често пъти акцентът само върху технологичните средства не води до промяна.

\section{Семантично значение на понятието "кибер"( 'cyber')}

В момента новите модели за водене на информационна и мрежова война, задължително включват това което е било актуално като направление през 90-те години, а именно компютьрни, мрежови операции(Computer Network Operations или CNO). Тези операции се провеждат с помощта на съвременни мрежи и са насочени срещу компютри, използвани за съхранение и обработка на информация. Случва се така, че в средата на 90-те години миналия век този етикет $\mathrm{CNO}$, имащ отношение към много специфични способности и действия се заменя с думата "кибер" в популярния дискурс. Това реално се случва след публикацията "Кибер войната настьпва", с автори Джон Аркуила и Дейвид Ронфелд, в академичното списание "Сравнителна стратегия"( Arquilla, and Ronfeldt,1993). Две години по-късно при провеждане на съвместно изследване с участие на няколко американски агенции за слабости в критичната национална инфраструктура на САЩ, възниква необходимостта от нова фраза, която да отговаря на предизвикателствата, свързани с уязвимостта на новите компютърни системи и се спират на думата "кибер" (Futter, 2018). Оттогава етикета "кибер",според разбиранията на Андрю Фътър (2018) се появява в практиката като опит да се замени понятията(IO/IW) информационни операции и информационна война, имащи отношение към нещо по-смислено като "компютьрно-мрежови операции" . Това се случва още поради факта, че новите компютърни технологии променят същността IO/IW, като се добавят в същото време 
нови ниши в концепцията за CNO. Този процес е свързан още със схващането за електронна война(Electronic Warfare), където новите дигитални способности налагат превключване в инфовойната и електронната война към значението на дигиталните технологии, отколкото към аналогови системи: и от мисията за прехващане и разрушаване на информационните потоци към нова цел: промяна, корумпиране и изтриване на информацията. Досега няма обща приета дефиниция (Futter, 2018) или концепция, която добре да обяснява фразите "суber” или “cyberthreat” (кибер и кибер заплаха). Факт е, че използването на тези термини обхваща широка област от различни сфери и сценарии, като се интегрира в изобилие от обекти и феномени. Съгласно професионалния речник в центъра на НАТО в Талин (Cooperative Cyber Defence Centre of Excellence) дефинират понятието "кибер" като непосредствено свързано с информационните технологии. Определението посочено в този речник е доста общо и може да означава различни неща. Терминът „кибернетика“ е позабравен, но отново популяризиран през 80-те години на 20. век като част от културата на движението и културата, свързан с "кибер пънк" (cyberpunk). Уйлям Гибсън (1984г), популярен американски писател в сферата на фантастиката, популяризира понятието „кибер“ в книгата си "Невромансър“. В края на 80-те години думата 'суber' се свързва с популярната култура, в частност с футуристичния стил и текстове от жанра на "антиутопия". По това време във военните крьгове на САЩ се обсъждат тезите, свързани с бойните действия в информационната епоха и използване на усъвършенствани компютърни системи за провеждане на военни операции, появяват се нови възможности за бойни действия. Като резултат възниква концепцията за информационна война, информационни операции и революция във военните отношения. Докато информацията става централна тема за войната, способностите да се определят като военна цел- комплексни компютърни системи, дигитални мрежи, електромагнитния спектър и медиите в реално време като част от информационна революция, позволява двете доктрини за IW/IO да се обогатяват и превърнат в специфично поле на военна дейност и стратегии.

\section{Характеристика на семантичната информация}

Информацията е разпозната като един от най-важните ресурси, които хората могат да използват ефективно, за увеличаване на стандарта за живот в информационната епоха. Клод Шанън (Shannon, 1948) дефинира важни елементи при анализа на терминологията: информация калкулирана от страна на източника, информационен капацитет на канала, кодиране и декодиране както на източника, така и на канала. Шанън допринася много и за развитие на комуникационната теория. Неговата теза е в по-голяма степен е свързана със синтактичния тип 
информация и малко отношение има към семантичната и прагматична част. Информационната теория на Шанън се разглежда като статическа теория на синтактичната информация, а не като теория на информацията като цяло. Освен тази теория има и други подобни тематични разработки, свързани с Алгоритмичната инфотеория и тази на Комбинаторната информационна теория. Класическата теория на семантичната информация започва с трудовете на Бар-Хилел и Карнап (Bar-Hillel and Carnap, 1953), като рамката и е разработена през 50-те години на миналия век. Тези двама учени предлагат идеален модел за език, свързан с този тип информация. Езикът съдържа следните важни символи: "n"-крайно положително число и "k" друго подобно число( двете числа представляват имена на съществителни). Допълнително се въвежда и един глагол под формата на "have, or be". Ако “a” е име на съществително и "Р" e прилагателно, и тогава “Ра” се смята като “а има качеството Р”, или “а е Р”. Модельт предложен от Зонг (Zhong, 2017) има пет важни връзки и могат да се генерират няколко предложения в логиката: истина(PaV Pa); лъжа (Pa^ Pa); неопределено((Рa).След което достигат до крайна формула $I(i)=-\log 2 m(i)$. В тази теория има два недостатъка: единият е свързан с идеалния модел на езика, койот е много далеч от естествения, реален език; втория- теорията има отношение само към количествени измервания на семантичната информация и не се интересува от есенцията/същността на информация и значението.

\section{Обобщаване на отделните концепции}

Обстоятелствената или пълна информация включва триадата синтактична, семантична и прагматична информация и се нарича всестранна, всеобхватна информация. Възприетата/изчерпваща информация се състои също от три компонента. Тези части на информацията са дефинирани в семиотиката, но не са изучени в достатъчна степен взаимоотношенията между тях. Синтактичната е специфична и може да се извлече от субекта посредством сетивните функции. Прагматичната се формира в зависимост от опита на субекта. Семантичната е абстрактна по природа и не може да се създава чрез сетивните органи. Може да се генерира като се използват едновременно синтактична и прагматичната, обединяване на двете и трансформиране в семантична, след това се дава наименованието и. От гледна точка на информационната екология трябва да се разгледат и ограниченията за семантичната информация. При този четвърти случай влияние оказват теориите на Шанън (Shannon, 1948), Винер (Wiener,1965) и Берталанфи (Bertalanffy, 1968). Последният разглежда информацията като важна характеристика на комплексността на системата. Знанието за решаване на проблеми се определя от интуицията, въображението, вдъхновението и естетичната нагласа. Зависи от 
способностите да придобиваме информация, да обработваме информация, разбиране, търсим смисъла и прилагането и. От тази гледна точка изкуственият интелект може да се определи като интелигентност, интегрирана от човека в машина, базирана на знание и е силно зависима от семантичен тип информация. Семантичната информация играе важна роля при конструиране на знание и изследване на интелигентността.

Известният британски учен Роджър Пенроуз (2020, с. 29- 30) смята, че ролята на компютьрните симулации, които играят в съвременната наука са базирани на вярата, че материалните обекти сами по себе си, в някакъв смисъл са просто "информационни структури", като подлежат на манипулиране чрез изчислителни, математически техники. Освен това квантовата теория изглежда ни казва, че материалните частици са просто "вълни" от информация. $\mathrm{R}$ или реалността е някакъв вид илюзия, трик или приближение и не следва да се приема като част от действителната еволюция на реалността, която следва да се опише на квантово ниво. Това предположение изглежда ни тласка към подходи за интерпретацията на Евърет или теорията за множествените светове. Когато говорим за изчисления се има предвид, че цялата структура на сигурността на информационните системи е ориентирана към точно изчисление на процесите, заплашващи съществуване на институциите., в смисъла, който този термин се отнася до съвременните дигитални компютри и техните теоретични предшественици-машините на Тюринг. Има и друг тип изчислителни(аналогови) устройства, които са използвани най-често в миналото и чието действие не се представя чрез дискретни състояния от типа "включено", "изключено", каквито са налице при дигиталните им наследници, а чрез непрекъснати физически параметри. По повод на аналоговите системи трябва да отчетем обстоятелството, че стандартните понятия за изчисления и изчислимост се отнасят, строго погледнато към дискретни системи(точно това означава дигитален), а не към непрекъснати системи, свързани с параметри като разстоянието или електричния потенциал. които имат отношение към класическите физически теории. Всъщност компютърни симулации на физически системи в общия случай апроксимират всички непрекъснати параметри посредством дискретни. Това обаче поражда грешки и при определен тип физични системи определено ниво на точност на апроксимация може да се окаже недостатъчно. В такива случаи дискретни компютърни симулации ще ни дадат погрешни заключения, относно поведението на непрекъснатата физическа система, която бива симулирана. Основна теза на Пенроуз ( 2020, c.41) имаща отношение към изкуствен интелект е, че „физичното функциониране на мозъка поражда съзнание и това функциониране не може да се симулира по адекватен начин чрез 
изчислителни процеси . Не поддаваща се на изчислително симулиране материална основа на феномена на осъзнаването би обяснила неизчислителната природа на "разбирането". Оттук следват следните зависимости: "интелект изисква "разбиране"; "разбиране" изисква "осъзнаване"

\section{Когнитивна реалност и компетентност}

От гледна точка на семантичните и когнитивни компетенции Еко (2004 с. 128) говори за двойна лексикална компетентност: инференциална компетентност и референциална. Според Еко вторият тип може да се раздели на три различни групи: инструкции за разпознаване; за идентификация; за намиране на обекта-особено важни функции при системите базирани на машинен интелект и платформите за автоматично визуализация на информация. Подобни компетенции би трябвало да се изградят при дефиниране на стратегии или архитектурата за инфо. Когнитивната среда на даден индивид е множеството от фактите, които му се явяват тази когнитивна среда заприличва много на това, което наричам КТ(когнитивен тип), и за да предположа наличието му у говорещия, и аз трябва да имам една репрезентация под формата на НС. Инференцията, договарянето се отнасят до усилието да се направят обществено съвместими нашите когнитивни среди, някои изненади пред гъвкавостта на нашите семиотични инструменти се пораждат от факта, че в почти всички дискусии за неуловимостта на значението, значението на термините се бърка със смисъла на изказването. Но проблемът не е само в това. Проблемът е също, че елементарните изказвания се бъркат с текстовете. Текстуалната семиотика отдавна вече признава, че може да се откриват системи от конвенции на граматично равнище и въпреки това да се приема, че на текстуално равнище стават договаряния. Ние може да не знаем изобщо какви свойства има получателят но знаем, че това име (адрес) посочва (чрез верига от електронни явления, които не е нужно да анализираме в подробности, но на чиято ефективност сме свидетели всеки ден) една индивидуална същност, която може да се различи от всяка друга, независимо от нашите вярвания, мнения, лексикални познания и от познанието, което имаме относно начина, по който името „посочва“ същността. Всички невъзможни обекти са немислими, но не всички немислими обекти са невъзможни. Еко смята, че когнитивния тип предполага четири прехода: стимули (това, което наричаме обекта сам по себе си); трансформация (извършената работа при дизайна); перцептивен модел; семантичен модел. От казаното дотук се вижда, че когнитивният тип би трябвало да изпълнява сега двойната функция на двата „модела“ —перцептивен и семантичен. Тунелното виждане се дефинира като тенденция за фокусиране на вниманието върху една цел или гледна точка. 
Подобна ситуация ограничава възможностите за перцепцията както на човека, така и на машината.

"Визията за тунела или тунелният ефект при когнитивна обработка на информацията е добре известно състояние в науката (Yujia, 2008). Този феномен в рискови ситуация означава огромно количество, противоречива информация и ограничено време за вземане на решение, което може да доведе до когнитивно претоварване. При стресови ситуации, каквито са атаките по мрежите, вземащите решения често са склонни към пристрастия, ниски равнища на разбиране на ситуацията и пропуски при решаване на проблеми. Проучванията показват, че двусмислена или противоречива информация лесно се игнорира, а подкрепящата и неутрална информация се използват за укрепване на първоначалните твърдения. След като се формира решение, мениджърите са склонни да се придържат към него, без да проучвате други възможности. Това може да доведе до повишено пристрастие в диагностика на реалната проблемна ситуация. Изправени пред крайна несигурност, хората са склонни да засилят търсенето на информация, едновременно става изключване на някои комуникационни канали, като се разчита на позната или официална информация или канали. Следователно, всеки сложен проблем, който изисква много информационни елементи и променливи краткосрочната памет може да допринесе за когнитивно претоварване. Информативно претоварване възниква, когато наличните когнитивни способности са недостатъчни, за да отговорят на изискванията изисквания за обработка. Тунелната визия (Yujia, 2008) в контекста на управлението на критични ситуации се определя като процес на създаване на рамка- пристрастия и предубеденост за потвърждение в когнитивните процеси на информационните мениджъри поради висок риск, висок стрес, голяма несигурност и висока плътност на информацията, причинени от кризисното събитие. От друга гледна точка в квантовите системи един от най-значимите процеси е така нареченото квантово тунелиране на електронните вълни през потенциална бариера. В съвременния свят важно приложение на квантови компютри е криптографията, в случаят, замисъльт е да се използва квантовото състояние за кодиране на информацията, като това гарантира много поголяма сигурност. Квантовата криптография използва един важен принцип: при условие че външна, враждебна страна се опита да разчете въведения квантов код, намесата й веднага ще бъде засечена. Квантовите системи позволяват защита на класическата информация от фалшифициране или неоторизиран достьп от външна страна, с недостатъчна степен на доверие.

\section{Изкуствен интелект-семантични проблеми}


В концепцията за „машинен човек“ Жак Боло (Bollo, 2016) отбелязва, че ИИ(изкуствен интелект) или роботиката са в същата ситуация като хората, що се отнася до информацията. Това е отричането на отразяващото съзнание в полза на един вид вродено намерение. Не трябва да се преструваме, че забравяме, че външното програмиране на машина, често ограничено до експериментални микросветове, също съответства на човешкото програмиране. В допълнение към външното програмиране на цялото обучение, противниците на ИИ отказват да позволят правилата да бъдат изрични, когато описват човек, отричайки критерия за средства / крайна връзка. От много конкретни примери може да се установи, че тази грешка се основава на дефектен учебен модел или реална среда. Решението на феноменологията, която претендира да обясни човешкото същество, е ограничено само от биологичния редукционизъм. Приложим е модельт на интелектуална измама-проекта Sokali. Случаят с програмата ELIZA на може да бъде преосмислен като успех на теста на Тюринг, въпреки че програмата допуска обезпокоителни грешки. В този модел на ELIZA, критиките към опоненти на ИИ могат да бъдат генерирани автоматично. Декомпилирането им е сравнително лесно да създаде пародия, която ги имитира (Dreyfy, Searly, Weizy, Winflory) като програма ELIZA. В крайна сметка това може да е истинският проблем на парадокса на новия актьор. Обичайните философски противоречия и отхвърлянето на неутралността пречат на анализа на когнитивните и комуникационни процеси. Автоматичната система може да отчита несъответствия. Компютьрните „техники“ като метода Merise (или „софтуерни работилници“) вече са автоматизирана процедура за формализиране на конкретни дейности.

Семантичният анализ, например, на стратегията „Кибер устойчива България(2020)”"1 показва някои специфики при формулировките и отделните, използвани термини. В науката за стратегията са известни няколко важни условия, при тяхното формулиране, дизайн и структура: уникалност( не се допуска цитиране на други автори при формулировката и- в горния документ има десетки цитати и позовавания); тотално превъзходство в съответната сфера(шансовете на конкурента за противодействие се свеждат до 0 ); концентрация на усилията в една важна точка, откъдето се контролира цялото пространство в конкретна област или домейн, в случая инфодомейна; име на стратегията(син океан, перли в короната, шок и ужас, red herring, Барбароса, D-Day, като в предложения стратегически документ липсват подобни понятийни и комбинации. В посочената стратегия графичната схема на отделните, административни

\footnotetext{
${ }^{1}$ Стратегия Киберустойчива България 2020 https://www.strategy.bg/StrategicDocuments/View.aspx?lang=bgBG\&Id=1120
} 
структура се нарича "модел", тоест архитектура на сигурността се свързва с обикновена схема. В текста понятието платформа се използва 6 пъти, но няма отношение към конкретна за целите на стратегията платформа. В случая със стратегията за "Киберустойчивост"(която по същество е административен документ, с малко допирни точки свързани с разбиранията за стратегия), прилича на десетки други приети в страната стратегии и разбира се нереализирани. Поадекватно наименование би било разработване на "Програма за тренинг и обучение на служители от националната критична мрежа, ориентирана към изграждане на мрежова, дигитална грамотност и сигурност на информационните системи"(Маринов 2021, с. 50-52.). Твърди се, че вече живеем и се развиваме в - кибер пространството. Кибер пространството е електронен или „виртуален“ свят от взаимосвързани комуникационни и информационни системи, в чиито мрежи глобалната общност е около 3 милиарда граждани. Всяка стратегия започва с мисия(липсва и намек за това ключово понятие(вместо това има Комисия). Тоест посочената стратегия е фиктивна, представлява имагинерен, административен документ и има малко отношение към съвременните разбирания за стратегии. Друг важен елемент от стратегиите е системен анализ-липсва каквато и да е дума в тази посока. Липсват и ключови думи като например за: виртуална реалност, големи данни, парсинг, структурирани данни, семантична информация; да не говорим за "кибернетика"; стандарти-28 пъти но не са дефинирани ясно/кои са); говори се и за съвременната "икономика на знанието", но липсва дума или намек за "трансфер на знание". Словосъчетанието „кибер пространство“ се среща 77 пъти в текста, честотата го обезсмисля като термин. Ако опитаме да си направим експеримент и игнорираме приставката „кибер“, използвана десетки пъти в документа, самата стратегия ще се изчерпи от съдържателна гледна точка. В тази връзка по-долу изброяваме няколко ключови дефиниции за кибер пространство, предложени от Гибсън, който въвежда основните понятия в тази специфична област 30 години преди този документ или в далечната 1984г. "Киберпространство, според Гибсън, е една съгласувана халюцинация, която ежедневно се изживява от милиардите, законно действащите оператори по целия свят. Едно графично представяне на информацията, която се извлича от базата данни на всички компютри в границите на човешката система. Немислимо по своята сложност. Поредица светлинни изображения на виртуалното пространство на съзнанието, могъщи струпвания и съзвездия от информация. За достьпа до това пространство се използва своеобразно устройство, наречено "кибердек" с помощта на което в пределите на това пространство се визуализират концентрични, всевъзможни хипнотични образи, подобно на филм, монтиран от случайно 
подбрани кадри. Символи, фигури, лица-размазан калейдоскоп от визуална информация. Киберпространството е драстично опростяване на човешката сензорна система. Мрежата характерна за това пространство се нарича Sense/Net или сетивна мрежа, със специална защита под формата на криогенна структура, приличаща на лед“ (Gibson,1996, c.59-60).

По-нататък изброявам някои от типичните елементи на семантичните компютърни (SC) технологии: структурирани и полу-структурирани данни; мултимедийни данни; текст; програми и услуги; мрежово поведение. Връзката между съдържание и ползвател се реализира с помощта на техниките: "семантичен анализ"; "семантична интеграция"-интегриране на съдържание и значения, извличани от многочислени източници; "семантични приложения"използване на семантики и съдържание за решаване на проблеми; "семантични интерфейси"опит да се интерпретира намерението на читателя, изразено с помощта на езика или с други форми на комуникация.

Посочвам и четири, важни компонента на общата методика, базирана на знание, за управление на критични процеси в съвременните мрежи: а/ данни; б/ информация(семантична); в/ интелигентни анализи; г/ знание(трансфер и управление- дизайн на КМ архитектури). Семантичната мрежа (Маринов, 2005, с.144) е нов тип структура, ориентирана главно към качествено съдържание на уеб ресурсите, създава се атмосфера, където софтуерни агенти скитащи от страница на страница имат готовност да отговорят на въпросите и запитванията на потребителя. Семантичната мрежа не е отделна мрежа, а разширява сега съществуващата, където информацията се трансформира и предава под формата на добре-структурирано съдържание. Необходимо е като първа стъпка при създаване на тази мрежа в близко бъдеще да се развият нови функции, така че машините да придобият по- големи възможности за обработване и разбиране на данните, които в момента се представят. Основната ценност на мрежата е нейната универсалност. Силата на хипертекстовите връзки е, че всичко може да се свърже с всичко. Не трябва да бъде дискриминирана или омаловажавана стойността на информацията. Сега информацията е изключително диференцирана и ограничена, т.е. има разлика между информация, предназначена за използване от хората и информация използвана от машините. Към днешна дата мрежата се е развила, по-скоро, като медиум за документи, предназначени за използване от хората, отколкото като хранилище за данни и съществена информация, изпълнявани по заявка на потребител автоматично. Семантичната мрежа е ориентирана именно в тази посока. Подобно на Интернет семантичната мрежа също трябва да бъде децентрализирана. По аналогия с другите мрежови технологии да генерира силни усещане 
на различни равнища, от големи корпорации до индивидуален потребител и да осигурява предимства и ползи, които трудно могат да се прогнозират предварително. За да функционира семантичната мрежа, компютрите би трябвало да имат достъп до колекция от структурирана информация и до мрежа от подразбиращи се правила, които да бъдат прилагани автоматично при получаване на знания в определена област. Изследователите на изкуствен интелект отдавна се занимават с тези проблеми. Knowldge representation както се нарича понякога семантичната технология и е сравнима с хипертекста и съществува още преди да бъде изобретена мрежата. Традиционната система за представяне на знание типично е централизирана, изисква всеки да споделя една и съща дефиниция за общи концепции. Контролът затруднява развитието и е неприложима за много дейности. Още повече при традиционната система въпросите, които могат да бъдат зададени са ограничени, това означава че повечето случаи и компютрите отговарят по принцип. Предизвикателство за семантичната мрежа е да се разработи език, които да може изразява данни и смислени правила за данните, което позволява правилата от съществуващи системи за представяне на знания да бъдат пренесени и в мрежата. Да се предаде логика на мрежата- средства за използване на правилата, за да се направи някаква връзка, да се избере направление за действие и да се отговори на въпросите. Логиката трябва да бъде достатъчно мощна и способна да описва комплексни черти на обектите, така че софтуерните агентите да могат отчитат и парадоксите на ситуацията.

Накрая се сприрам на характеристиките на една семантична, изчислителна система, наречена SIR -Semantic information retrieval. Това е една от първите динамични, компютьрни системи за извличане на информация и структурирането и съгласно, базови семантични принципи. Системата е добре описана от един от създателите на системите за изкуствен интелект Марвин Мински (Minsky,1968, p. 32-42). SIR системата използва специална структура на данните, наречен модел. Програмата се обръща или взаимодейства с модела, когато трябва да съхрани или извлече семантична информация. Програмата е ориентирана към решаване на проблеми или това е вторият използван модел за дефиниране на сьщността извлечената информация. Третият модел интегриран в интелигентната система е ориентиран към "въпросотговор". Информационното съдържание е свързано със семантичното съдържание и няма отношение към количеството съхранени битове. Информацията се съхранява в специални места на системата, моделът е базиран само на ясно, дефинирани изречения на английски език и се извлича от мрежите по строго определен начин. Програмата класифицира всички думи като функционални думи- които притежават структурно и синтактично значение и думи- 
ориентирани към съдържание, които притежават семантична стойност и свързаност. Основата на подобни системи са енциклопедиите, които се респектират от думите със значение. Интелигентните системи изпълняват ясно дефинирани специфични задачи. Това още е динамичен модел, в смисъл че нова информация може да предизвиква автоматично добавяне на данни и промени в структурата им, В допълнение това е и семантичен модел, в смисъл че данните са организирани в структури, които представят значението на изречения, базирани на английски. Друг, популярен модел за семантично извличане на информация е базиран на асоциации между думи, това е коренно различен подход и подобна система се отличава с поголяма интелигентност и може да имитира човешко поведение. В системата още е интегрирана математическа логика. SIR работи със словесни асоциации, представени под формата на първоначални описания, като търси съответствия и взаимоотношения между отделните думи. SIR е конструирана така че да взаимодейства с хората, последните да могат да задават въпроси и получават отговори на естествен език. Целта е системата да съхранява и използва по рационален начин информацията, за да осигури си впоследствие интелигентно поведение. а да се изгради обща система за извличане на семантично, вързана информация се изискват и точно дефинирани проблеми, за съжаление голяма чат от комплексните проблеми днес нямат ясна формулировка.

\section{Заключение}

В съвременните общества е необходимо мениджърите по защита на информацията да отчитат семантичните и аспекти и след това да пристьпят към оценка важността на информационните ресурси, които ще защитават и там да съсредоточат своите усилия, като се осигурят технологични и превантивни мерки. Когнитивните, аналогови и квантови системи ще играят все по-значима роля за противодействие на атаките, насочени към информационния домейн и успешната му защита.

\section{Използвана литература}

Arquilla, J., \& Ronfeldt, D. (1993/2007). Cyberwar is coming! Comparative Strategy, 12(2), 141165, published online 2007. https://doi.org/10.1080/01495939308402915

American Society of Cibernetics (ASC). (https://asc-cybernetics.org).

Bar-Hillel, Y., \& Carnap, R. (1953). Semantic information. British Journal for the Philosophy of Science, 4, 147-157. https://doi.org/10.1093/bjps/IV.14.147

Bertalanffy, L. (1968).General System Theory. New York, GEORGE BRAZILLER 
Bolo, J. (2016, June). Philosophie contre intelligence artificielle [Philosophy versus artificial intelligence]. http://www.exergue.com/h/2016-06/tt/philosophie-ia.html

Davenport, T. (1997). Information ecology. Mastering the information and knowledge environment. Oxford University Press.

Eko, U. (2004). Kant i ptitsechovkata [Kant and the platypus]. Sofia, Izd-vo Dom na naukite za choveka i obshtestvoto. e-pub https://biblioman.chitanka.info/books/7883

Futter, A. (2018). Cyber' semantics: why we should retire the latest buzzword in security studies, Journal of Cyber Policy, 3(2), 201-216. DOI: 10.1080/23738871.2018.1514417, 2018.

Gibson, W. (1996). Nevromantic [Neuromancer]. Sofia, Izd-vo Kamea. epub format https://chitanka.info/book/482-nevromantik

Marinov, R. (2021). Krizi i prvencia [Crises and prevention]. Izd-vo Avangard Prima.

Marinov, R. (2005). Stratrgicheski komunikazionen menidjmunt [Strategic communication management]. Sofia, Izd-vo Siela.

Penrose, R. (2020). Senkite na uma [Shadows of the Mind]. Izd-vo Iztok I Zapad

Minsky, M. (1968). Semantic information processing. The MIT Press.

Shannon, C. E. (1948). A mathematical theory of communication reprinted with corrections from the bell. System Technical Journal, 27, 379-423; 623-656.

Wiener, N. (1965). Cybernetics or control and communication in the animal and the machine. (2nd ed.). The MIT press.

Yujia, C. (2008). An information assistant system for the prevention of tunnel vision in crisis management. Computer Science Journal of Scheduling. Corpus ID: 15353483. https://bit.ly/3D2xBQt

Zhong, Y. (2017, June 17). A theory of semantic information. [Presentation at the IS4SI 2017 Summit Digitalization for a sustainable society, Gothenburg, Sweden, 12-16 June 2017]. https://sciforum.net/manuscripts/4000/manuscript.pdf. 